\title{
Satisfacción laboral: el camino entre el crecimiento psicológico y el desempeño laboral en empresas colombianas industriales y de servicios*
}

\section{Job Satisfaction: The Way between Psychological Growth and Job Performance in Industrial and Service Companies in Colombia}

Recibido: julio 22 de 2012 | Revisado: enero 21 de 2013 | Aceptado: marzo 28 de 2013

\author{
John Alejandro Sanín Posada** \\ Centro de Investigación en Comportamiento \\ Organizacional, Colombia \\ MARISA SALANOVA SORIA *** \\ Universidad Jaume I, Castellón, España
}

doi:10.11144/Javeriana.UPSY13-1.slcp

Para citar este artículo: Sanín, J. A. \& Salanova, M. (2014). Satisfacción laboral: el camino entre el crecimiento psicológico y el desempeño laboral en empresas colombianas industriales y de servicios. Universitas Psychologica, 13(1), 95-107. doi:10.11144/ Javeriana.UPSY13-1.slcp

* Artículo de investigación.

*** Centro de Investigación en Comportamiento Organizacional - CINCEL. Colombia (http://www. cincel.com.co).E-mail: asaninp@cincel.com.co

**** Work and Organizational Network (WONT), Prevenció Psicosocial (http://www.wont.uji.es). Directora del Doctorado en Psicología del Trabajo y de las Organizaciones, Universidad Jaume I, España. E-mail: marisa.salanova@uji.es

\section{RES UMEN}

En el presente estudio se analiza cómo la satisfacción laboral media las relaciones entre el crecimiento psicológico (apertura al cambio, manejo del fracaso y flexibilidad) y el desempeño laboral (extrarrol, intrarrol y cumplimiento de normas), evaluado este último por los jefes. Participaron 731 empleados y sus correspondientes jefes de cinco empresas colombianas de los sectores industrial y de servicios. Los resultados obtenidos a través de Ecuaciones Estructurales (Structural Equation Modeling, SEM) muestran que la satisfacción laboral media parcialmente la relación entre apertura al cambio y desempeño extrarrol (evaluado por el jefe). Se encontró además que la flexibilidad y la apertura al cambio se asocian positiva y directamente con la satisfacción laboral general y que esta, a su vez, predice el desempeño extrarrol y el cumplimiento de normas, evaluados por el jefe.

Palabras clave autores

Crecimiento psicológico, satisfacción laboral general, desempeño laboral.

Palabras clave descriptores

Psicología organizacional, calidad de vida, Colombia.

\footnotetext{
A B S T R A C T

We analyze the relationship among psychological growth (Openness to Change, Management of failure and Flexibility) and job performance (extrarrol, intrarrol and Compliance), mediated by overall job satisfaction. This study used a sample of 731 employees and their immediate boss of five Colombian companies from the industrial and service sectors. The results using structural equation (SEM) show that job satisfaction partially mediates between openness to change and extrarrol performance (assessed by the immediate boss). Flexibility and openness to change are positively and directly associated with overall job satisfaction and this predicts extrarrol performance and compliance, assessed by the boss.

Keywords authors

Psychological growth, overall job satisfaction, job performance.

Keywords plus

Organizational psychology, quality of life, Colombia.
} 


\section{Introducción}

El hecho de que el desempeño laboral afecte la productividad de las organizaciones (Toro, 2000) ha llevado a que los investigadores quieran determinar sus antecedentes y consecuencias. En la literatura, se pueden hallar numerosos modelos que intentan cumplir con este propósito (Muchinsky, 2002), y que evidencian aspectos que lo afectan directa o indirectamente (Sanín \& Restrepo, 2009). Recientemente, se ha desarrollado en Colombia un modelo de factores psicosociales que permite entender la complejidad de las relaciones existentes entre las dimensiones organizacionales, las individuales, los resultados de esta interacción y sus efectos sobre la salud, el bienestar, el desarrollo y, por supuesto, el desempeño de las personas en el trabajo (Toro, Londoño, Sanín \& Valencia, 2010). Dentro de las variables que integra este modelo se encuentran el crecimiento psicológico (en adelante CPs), la satisfacción laboral y el desempeño, donde la primera variables se muestra como una condición antecedente de las dos siguientes.

La relevancia de estos temas para la psicología organizacional y para las empresas radica en comprender que el comportamiento de las personas, es decir su desempeño, es un fenómeno psicológico que determina la productividad (Toro, 2002), por su parte, la satisfacción favorece el deseo de permanencia de las personas y se asocia con la reducción del ausentismo, la rotación y las quejas del personal (Littlewood, 2008). Se trata, por tanto, de temas de interés empresarial en tanto apuntan a la solidez, eficiencia y permanencia organizacional, lo cual es fundamental en un contexto competitivo y globalizado como el que enfrentan las organizaciones actuales, no estando exentas las colombianas de esta demanda global. Al tratarse de temas de carácter psicosocial, es imperativo que los investigadores de la Psicología del Trabajo y las Organizaciones asuman el reto de explorar las condiciones psicológicas y sociales que moderan o favorecen la eficiencia de las personas y por tanto la productividad de la organización.

El CPs es una variable psicológica que puede afectar el comportamiento de las personas en el trabajo y su satisfacción (Romero, 1999), de ahí el interés de analizarlo. Este trabajo se centrará en la exploración de las relaciones entre este constructo, la satisfacción laboral general y el desempeño laboral, siendo la satisfacción el mecanismo psicológico que explicaría la relación entre crecimiento psicológico y desempeño, esto es, que ejerce un rol mediador. Se entiende pues que la apertura al cambio, el manejo del fracaso y la flexibilidad (dimensiones de $\mathrm{CPs}$ ) favorecen evaluaciones más positivas sobre la realidad y aportan, por ello, a la satisfacción del personal (Romero, 1994), la cual, a su vez, puede influir sobre el desempeño de las personas (Zelenski, Murphy \& Jenkins, 2008).

El crecimiento psicológico se ha definido como un proceso a través del cual las personas generan estructuras cognitivas que les permiten integrar su realidad exterior e interior (Romero, 1999). Se refiere a la forma como se interpreta la realidad para conseguir visiones más positivas sobre el mundo. Implica un manejo abierto y creativo de la incertidumbre, una amplia apertura al cambio, flexibilidad y manejo del fracaso desde un punto de vista positivo como una importante oportunidad de aprendizaje (Eljuri, 1994; Romero, 1994). Si bien se le reconoce como proceso, la aproximación a este concepto suele hacerse a través de la exploración del nivel en el que se encuentra un conjunto de variables (dimensiones) que lo facilitan.

Inicialmente el CPs, como factor de segundo orden, se operacionalizaba a partir de cuatro dimensiones: apertura al cambio, flexibilidad, manejo de la incertidumbre y manejo del fracaso (Romero, 1994). Sin embargo, en Colombia un estudio reciente mostró una estructura diferente, conformada por tres dimensiones en donde flexibilidad y manejo de la incertidumbre se agrupan en un mismo factor. Apertura al cambio y manejo del fracaso permanecieron como independientes (Sanín, 2010).

La apertura al cambio se entiende como la "percepción de la persona sobre su disposición para exponerse a experiencias nuevas y crecer a partir de ellas" (Sanín, 2010, p. 150). Se trata de la facilidad que ve de afrontar situaciones nuevas, de su juicio sobre la conducta que suele mostrar ante el cambio. 
La flexibilidad, por su parte, se conceptualiza como la posibilidad que tienen las personas de generar cambios que les permiten adaptarse (Kaiser $\&$ DeVries, 2010). Caracteriza a personas con iniciativa, que manejan la incertidumbre de manera adecuada y que logran juzgar su entorno desde diversos puntos de vista (Elaine, Pulakos, Arad, Donovan \& Plamondon, 2000). Se operacionaliza como el "grado en que la persona se considera capaz de generar construcciones alternativas de personas o situaciones que le ayudan a crecer" (Sanín, 2010, p. 150). Se diferencia de la apertura al cambio en tanto implica una trasformación que puede ser observada. La apertura solo habla de la disposición.

Finalmente, el manejo del fracaso es la posibilidad de ver positivamente los errores, entenderlos como oportunidades de aprendizaje (Newton, Khanna \& Thompson, 2008). Esta dimensión se operacionaliza como "el nivel en que la persona se considera capaz de utilizar información presente de las situaciones de fracaso de manera favorable para su crecimiento personal" (Sanín, 2010, p. 150). Constituye un punto fundamental del CPs en la medida en que, desde una connotación positiva, permite crecer a partir del aprendizaje que se obtiene del error.

\section{Crecimiento Psicológico y Satisfacción Laboral}

Las satisfacción laboral se entiende como una reacción afectiva que surge al contrastar la realidad laboral con las expectativas relacionados con esa realidad (Wright \& Cropanzano, 2000). En este trabajo, se operacionaliza como el nivel de complacencia o agrado que la persona experimenta en relación con su realidad laboral, y se refiere a la reacción afectiva con el trabajo visto como un todo, sin contemplar aspectos particulares de esta realidad (Wright \& Cropanzano, 2000). La investigación sugiere que su relación con el CPs podría deberse a que ambas son condiciones necesarias para el bienestar (Romero, 1994) y al hecho de que el CPs está asociado con la orientación de las personas hacia metas valiosas (Romero, 1994). Si la persona le concede valor a su satisfacción, diri- girá su proceso de crecimiento en esa dirección. También es posible pensar en la relación de estas dos variables cuando se analizan las dimensiones de CPs separadamente. Se ha encontrado que bajos niveles de apertura al cambio se relacionan con insatisfacción, irritación laboral y aparición de intenciones de renuncia (Wanberg \& Banas, 2000). Desde un enfoque positivo, cabe esperar que tenga una relación positiva con la satisfacción.

La flexibilidad, por su parte, favorece la adaptación, lo cual puede consistir en generar cambios que permitan obtener mayor satisfacción (Bond, Flaxman \& Bunce, 2008). En cuanto al manejo del fracaso, se sabe que cuando se tiene una visión positiva sobre este las personas pueden sentirse más complacidas con las realidades que los rodean (Morales de Romero, 2008); adicionalmente, saber manejar el fracaso se asocia con niveles más altos de autoestima (Shelley \& Silvia, 2002), la cual, a su vez, se relaciona con armonía, estabilidad emocional y autoeficacia, y negativamente con desajustes emocionales, improductividad y dependencia (Judge \& Bono, 2001).

En conjunto, la apertura al cambio permite una mayor flexibilidad en las expectativas (Wanberg \& Banas, 2000). La flexibilidad facilita modificar las realidades del trabajo que resultan adversas (Bond et al., 2008) y el manejo del fracaso contribuye a sacar provecho de las fallas y, al hacerlo, puede promover reacciones afectivas placenteras y satisfacción laboral (Shelley \& Silvia, 2002).

\section{Crecimiento Psicológico y Desempeño Laboral}

El desempeño se entiende como la acción o acciones que una persona realiza con el ánimo de obtener un resultado (Toro, 2002). En el trabajo puede incluir conductas orientadas al cumplimiento de las responsabilidades del cargo y al desarrollo de procesos inherentes a este (desempeño intrarrol), la realización de actividades extras que agregan valor (desempeño extrarrol) o a actuaciones coherentes con las normas y principios definidos por la organización (cumplimiento de normas) (Toro, 2010). Esas son las tres dimensiones de desempeño laboral que 
se analizan como variables dependientes en nuestro modelo. Su relación con el CPs puede explicarse en la medida en que la apertura al cambio, la flexibilidad y el manejo del fracaso contribuyen a la modificación de comportamientos (Romero, 1994). También porque el CPs se encuentra relacionado con el motivo de logro (Salom \& Romero, 1994), el cual implica un interés permanente de las personas por alcanzar metas retadoras, las cuales favorecen la persistencia y el esfuerzo y, por tanto, un mejor desempeño (Sanín \& Restrepo, 2009).

También es posible entender la relación entre estos constructos, si se analizan por separado las dimensiones del CPs. La apertura al cambio y la flexibilidad favorecen la adaptación de la persona, lo cual puede consistir en hacer el trabajo de la mejor manera posible, cumpliendo con las normas de la organización y agregando valor a la misma. Por su parte, una visión positiva del fracaso permite aprendizaje y desarrollo; esto siempre y cuando se valore como algo que enseña, como parte del proceso y no como el punto final (Polivy \& Herman, 2002).

De acuerdo con lo anterior y teniendo presente que se quiere probar un modelo de mediación, se parte de tres supuestos necesarios para confirmar la hipótesis acerca de que la Satisfacción Laboral media la relación entre el CPs y el Desempeño Laboral: 1) las variables independientes (Dimensiones de CPs) correlacionarán con la mediadora (Satisfacción Laboral) y con las dependientes (dimensiones de Desempeño Laboral). 2). la Satisfacción Laboral y el Desempeño correlacionarán positivamente y 3) al incluir la variable mediadora en el modelo, el efecto entre las variables independientes y la dependiente dejará de ser significativo.

\section{Método}

\section{Participantes y procedimiento}

En el estudio participaron 731 empleados de las principales ciudades de Colombia (Medellín, Bogotá y Cali), de diferentes edades (entre 21 y 25 años, 23.4\%; de 26 a 30 años, 22.6\%; de 31 a 35 años, 15.2\%; de 36 a 40 años, $12.7 \%$; de 41 a 45 años, 12.5\%; de 46 a 50 años, $5.8 \%$; de 51 a 55 años, 3.6\%; de 56 a 60 años, 1.2\% y más de 61 años, 0.1\%), áreas ocupacionales (Operario, 80.7\%; Profesional, 7.3\%; Empleado de Oficina, 6\%; Técnicos y Tecnólogos, 3.7\%; Directivos, $1.6 \%$ y vendedores $0.7 \%$ ), estratos socioeconómicos (estrato 2, $51 \%$; estrato 3, 25.5\%; estrato 1, 15.2\%; Estrato 4, 5.5\%; estrato 5, 1.7\% y estrato 6, 0.7\%), niveles educativos (Bachillerato, 66\%; Técnico Tecnológico, 16.6\%; Primaria, 7.9\%; Profesional, $7.1 \%$ y Posgrado, $2.3 \%$ ) y género (64.2\% hombres) y sus respectivos jefes. Encuestadores profesionales estuvieron a cargo de la aplicación de las encuestas y control de la información, siguiendo procesos de recolección estandarizados y normalizados, que se hizo a partir de encuestas aplicadas en grupos de entre 10 y 20 participantes. Los instrumentos se cumplimentaron en un promedio de 30 minutos. En la evaluación hecha por los jefes se les comunicó a quién o a quiénes debían evaluar. Cada persona fue identificada con un código, de tal forma que las evaluaciones hechas por sus jefes pudieran ser emparejadas con las que ellas habían hecho, respetando la confidencialidad. Cada jefe tardó alrededor de $10 \mathrm{mi}$ nutos juzgando el desempeño de cada uno de sus colaboradores. En todas las evaluaciones se pidió el consentimiento informado para la participación en el estudio.

\section{Instrumentos}

El CPs fue evaluado con la Escala de Desarrollo Psicológico (EDEPSI) (Sanín, 2010). Apertura al cambio fue medida con una subescala de cinco ítems $(\alpha=0.8)$ (e. g. de ítem: "Me interesa iniciar actividades nuevas con el fin de incrementar mis conocimientos"). Manejo del fracaso con una subescala también de cinco ítems $(\alpha=0.8)$ (e.g. de ítem: "Veo el fracaso como una posibilidad de desarrollar mis habilidades"). Flexibilidad se examinó con una escala de cinco ítems $(\alpha=0.7)$ (e. g. de ítem: "Enfrentar situaciones de las que no sé qué esperar me ha servido para desarrollar mis habilidades"). Los participantes respondieron con base en una escala tipo Likert de 6 opciones, siendo 6 (completamente de acuerdo) y 1 (completamente en desacuerdo). 
La satisfacción laboral general se midió con la Escala General de Satisfacción Laboral (EGSL) (Toro, 2010). Un instrumento de siete ítems ( $\alpha=$ 0.83) (e. g. de ítem: "En relación con las exigencias físicas de mi trabajo yo me siento..."). Los participantes respondieron con base en una escala tipo Likert de 6 opciones que van desde 6 (muy satisfecho) a 1 (muy descontento).

Las tres dimensiones de desempeño laboral fueron evaluadas con la Escala de Evaluación el Desempeño (EED) (Toro, 2010) por parte de los jefes. Desempeño intrarrol fue medido con una escala de cinco ítems $(\alpha=0.93)$ (e. g. de ítem: "Realizó todas las actividades especificadas para su cargo."). Desempeño extrarrol se midió con una escala de cuatro ítems ( $\alpha=0.85)$ (e. g. de ítem: "Asumió riesgos sin necesidad."). Cumplimiento de normas se examinó con una escala de cuatro ítems también ( $\alpha=0.84)$ (e. g. de ítem: "Cumplió las normas especificadas en el reglamento interno de trabajo"). Los jefes respondieron con base en una escala de 6 opciones que evalúa con qué frecuencia el empleado realiza conductas intrarrol, extrarrol y asociadas al cumplimiento de normas. La escala va desde 6 (todo el tiempo lo hace) a 1 (nunca lo hace).

Tanto las dimensiones de CPs como las de desempeño corresponden a variables latentes en nuestro modelo de ecuaciones estructurales. El CPs es un factor de segundo orden, sin embargo no se trabajó con este, en tanto se consideró que trabajar con las dimensiones permitiría una mejor comprensión del proceso psicológico y del tipo y la intensidad de las relaciones con la variable mediadora y las dependientes.

\section{Análisis de datos}

Se verificaron dos criterios de calidad psicométrica: la validez y confiabilidad de las escalas; la primera se examinó a través de análisis factoriales confirmatorios y la segunda con el alfa de Cronbach. Luego, para poner a prueba el sesgo de método de la varianza común se aplicó la prueba de un solo factor de Harman (Podsakoff, Mackenzie, Lee \& Podsakoff, 2003) con análisis factorial confirmatorio (CFA) para las variables de CPs y desempeño laboral (ver en la Tabla 2: M2 y M5m, respectivamente). Después, se realizaron análisis de ecuaciones estructurales utilizando el programa AMOS 16.0 para poner a prueba la hipótesis. Se verificó que se cumplieran los supuestos de la mediación, a saber: que cada una de las variables independientes se relacionara significativamente con la dependiente y con la mediadora y que, a su vez, esta lo hiciera con la dependiente. Luego, que al introducir la variable mediadora en la ecuación desapareciera el efecto de las independientes sobre las dependientes (Baron \& Kenny, 1986). De ser así se hablaría de mediación total. Si el efecto permanece, sería mediación parcial.

Se verificó el ajuste de cada modelo a través de la estimación de máxima verosimilitud. La bondad de ajuste se evaluó a través de los índices: Root Mean Square Error of Approximation (RMSEA); en este, valores inferiores a 0.07 se consideran aceptables en cuanto al nivel de ajuste, siempre que su probabilidad sea superior a 0.05; Normed Fit Index (NFI), Tucker Lewis Index (TLI) y el Comparative Fit Index (CFI) (Jöreskog \& Sörbom, 1986), en estos, valores superiores a 0.9 son considerados aceptables. El ajuste será mejor entre más cercano a 1 sea el valor. También se utilizó el índice chi cuadrado.

Se calcularon además los promedios, desviaciones típicas y correlaciones entre variables. No fue posible calcular el ICC (Intraclass Correlation Coefficient), lo cual hubiera permitido ver la adecuación de la medida de cada jefe a su grupo de colaboradores (McGraw \& Wong, 1996), debido a que la segmentación de los grupos de colaboradores fue en áreas mayores y no en pequeños grupos, con lo que fue imposible saber qué colaboradores compartían jefe.

\section{Resultados}

Se aprecian en la Tabla 1 las medias, desviaciones estándar y correlaciones entres las variables del estudio. Allí puede verse también el nivel de consistencia de cada escala.

Flexibilidad y manejo del fracaso mostraron correlaciones significativas entre ellas, sin embargo, solo apertura al cambio correlacionó con una de las 
TABLA 1

Correlaciones entre las variables del estudio, medias (M) y desviaciones estándar (DE)

\begin{tabular}{lllllllllll}
\hline & $\alpha$ & $M$ & $D E$ & 1 & 2 & 3 & 4 & 5 & 6 & 7 \\
\hline 1. APERTURA AL CAMBIO & 0.80 & 3.57 & 1.34 & - & & & & & & \\
2. FLEXIBILIDAD & 0.70 & 4.94 & 0.80 & 0.059 & - & & & & \\
3. MANEJO DEL FRACASO & 0.80 & 4.74 & 1.17 & 0.01 & $0.35^{* *}$ & - & & & \\
4. SATISFACCIÓN & 0.79 & 5.27 & 0.67 & $0.10^{* * *}$ & $0.16^{* *}$ & $0.08^{*}$ & - & & \\
5. DESEMPEÑO INTRARROL & 0.92 & 5.18 & 0.74 & 0.02 & -0.02 & 0.03 & $0.13^{* * *}$ & - & \\
6. DESEMPEÑO EXTRARROL & 0.94 & 4.20 & 1.71 & $0.34^{* *}$ & 0.02 & 0.05 & $0.12^{* * *}$ & $0.16^{* *}$ & \\
7. CUMPLIMIENTO DE NORMAS & 0.83 & 5.35 & 0.66 & 0.06 & -0.03 & 0.00 & $0.14^{* *}$ & $0.56^{* *}$ & $0.22^{* *}$ \\
\hline
\end{tabular}

Nota. Correlaciones: $* * p<0.01 * p<0.05$.

Fuente: elaboración propia.

TABLA 2

Ajuste de la estructura factorial de las escalas

\begin{tabular}{lcccccccccccc}
\hline & $\chi^{2}$ & $g l$ & RMSEA & $\mathrm{p}$ & NFI & TLI & CFI & $\chi^{2}$ & RMSEA & NFI & TLI & CFI \\
\hline M1 & 194.4 & 83 & 0.03 & 1 & 0.95 & 0.96 & 0.97 & & & & & \\
M2 & 1973.8 & 90 & 0.14 & 0 & 0.51 & 0.45 & 0.53 & & & & & \\
Diferencia & & & & & & & & $=1778.57$ & 0.11 & 0.44 & 0.51 & 0.44 \\
entre M2-M1 & & & & & & & & & & & & \\
M3 & 65.75 & 28 & 0.05 & 0.385 & 0.96 & 0.96 & 0.97 & & & & & \\
M4 & 243.2 & 58 & 0.05 & 0.205 & 0.97 & 0.98 & 0.98 & & & & & \\
M5 & 5289.4 & 65 & 0.27 & 0 & 0.53 & 0.43 & 0.53 & & & & & \\
Diferencia & & & & & & & & $=5046.19$ & 0.22 & 0.44 & 0.55 & 0.45 \\
entre M5-M4 & & & & & & & & $=50.45$ \\
\hline
\end{tabular}

Nota. $\chi^{2}=$ chi cuadrado; $g l=$ grados de libertad; RMSEA = Root Mean Square Error of Approximation;

$p=$ probabilidad de RMSEA; NFI = Normed Fit Index; TLI = Tucker Lewis Index; CFI = Comparative Fit Index.

M1 = Modelo de tres factores de crecimiento psicológico

M2 = Modelo de un factor de crecimiento psicológico

M3 = Modelo de un factor de satisfacción laboral

M4 = Modelo de tres factores de desempeño laboral

M5 = Modelo de un factor de desempeño laboral

Fuente: elaboración propia.

dimensiones de desempeño (extrarrol). Satisfacción laboral correlacionó con todas las dimensiones de CPs y de desempeño.

A través de ecuaciones estructurales se sometió a verificación el modelo de tres factores de CPs, el de un factor de satisfacción laboral general y el de tres factores de desempeño laboral. Luego, para poner a prueba el sesgo de la varianza común del método, se aplicó la prueba de un solo factor de Harman (Podsakoff et al., 2003), con análisis factorial confirmatorio (CFA) para las variables de CPs y desempeño laboral (Tabla 2). Se observa que los modelos de un solo factor de CPs (Delta $\left.\chi^{2}=1778.57, p<0.005\right)$ y de desempeño laboral (Delta $\chi^{2}=5046.19, p<$ 0.005) muestran un ajuste más pobre.

Luego se verificaron las asociaciones entre las variables, usando ecuaciones estructurales, para finalmente examinar el modelo de investigación. La Tabla 3 muestra los resultados.

Puede verse que los datos ajustaron bien en cada uno de los pasos. A continuación, se presentan los estimados y sus niveles de significación, a través de diagramas de ecuaciones estructurales; estos resumen los pasos seguidos para la verificación del modelo de investigación. 
TABLA 3

Pasos y ajuste del modelo de investigación

\begin{tabular}{lccccccc}
\hline & $\chi^{2}$ & $\mathrm{gl}$ & RMSEA & $\mathrm{p}$ & NFI & TLI & CFI \\
\hline M1 & 33.689 & 14 & 0.04 & 0.675 & 0.97 & 0.96 & 0.98 \\
M2 & 49.575 & 39 & 0.02 & 1 & 0.99 & 0.99 & 0.99 \\
M3 & 15.024 & 14 & 0.01 & 0.996 & 0.99 & 0.99 & 1 \\
M4 & 84.503 & 56 & 0.03 & 1 & 0.98 & 0.99 & 0.99 \\
\hline
\end{tabular}

Nota $\chi^{2}=$ chi-cuadrado; $g l=$ grados de libertad; RMSEA = Root Mean Square Error of Approximation; $p=$ probabilidad de RMSEA; NFI = Normed Fit Index; TLI = Tucker Lewis Index; CFI = Comparative Fit Index.

M1 = Modelo de la relación entre las dimensiones de crecimiento psicológico y la satisfacción laboral, M2= Modelo de relación entre las dimensiones de crecimiento psicológico y las de desempeño laboral, M3= Modelo de relación entre Satisfación Laboral y las Dimensiones de desempeño laboral, M4=Modelo de investigación.

Fuente: elaboración propia.

Se aprecia, primero, la relación entre las variables independientes y las dependientes (Figura 1). Los resultados evidencian que solo apertura al cambio se relaciona con uno de los componentes del desempeño, a saber con el extrarrol, lo que da soporte parcial al modelo.

Si bien esto sugiere la idea de que la satisfacción solo podría ejercer un rol mediador entre apertura al cambio y las conductas extrarrol, se mantuvo el

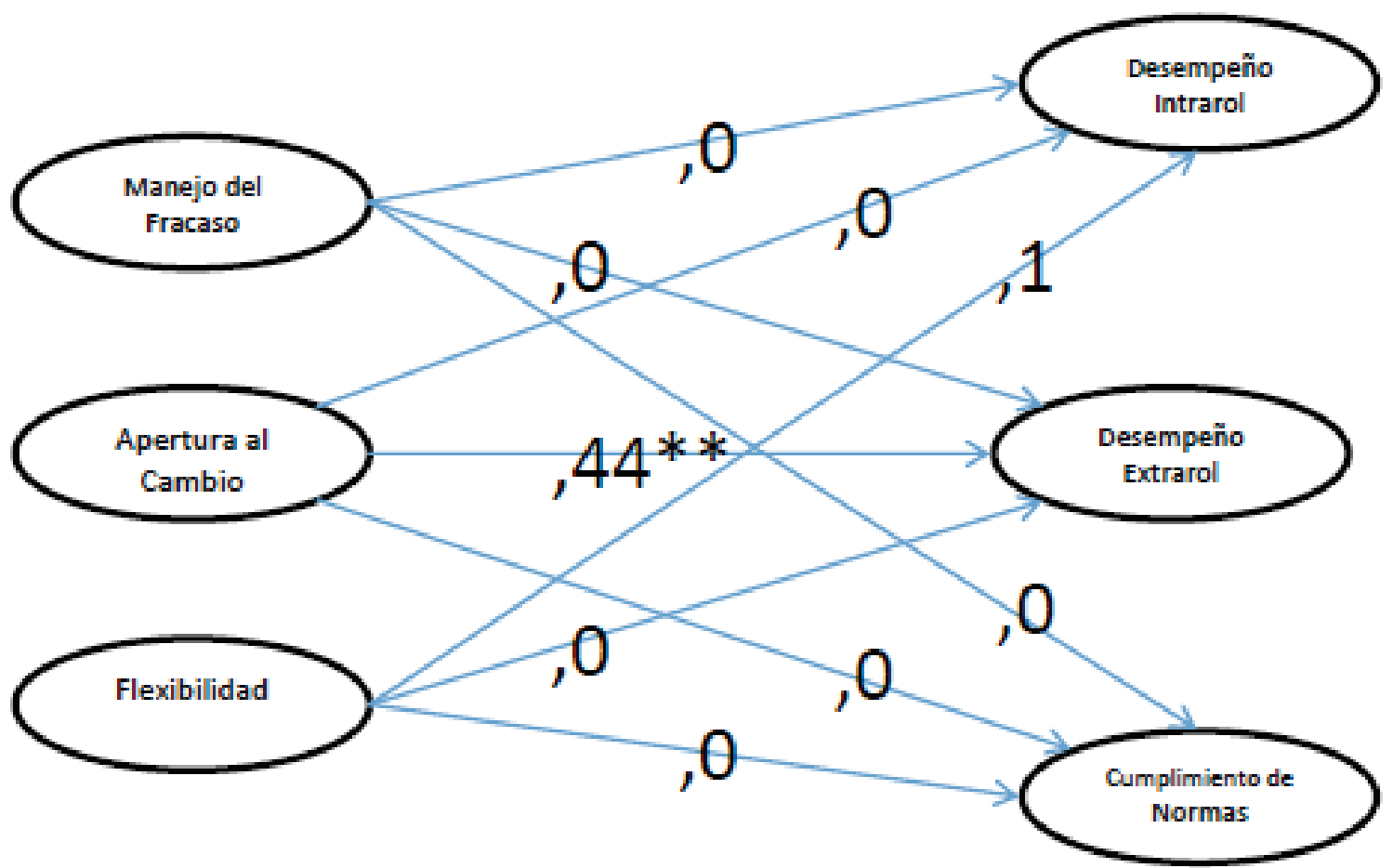

Figura 1. Significación y estimados estandarizados de las relaciones de las Dimensiones de CPs con las de desempeño laboral. ***p $<0.001$.

Fuente: elaboración propia. 


\section{Empleados}

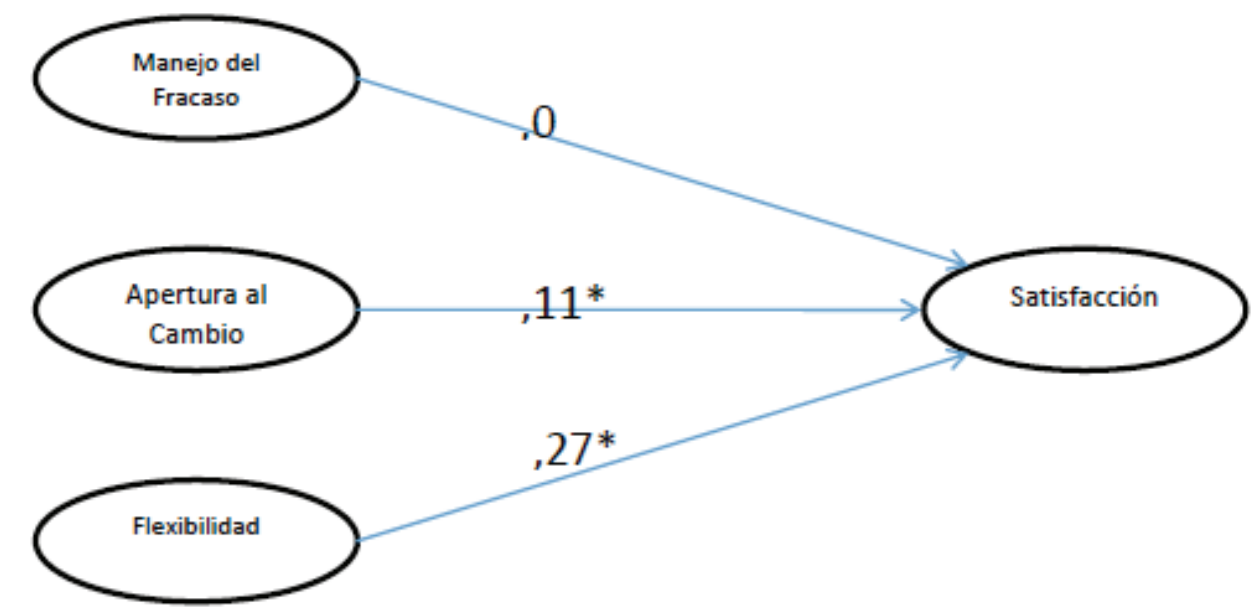

Figura 2. Significación y estimados estandarizados de las relaciones de las Dimensiones de crecimiento psicológico con la satisfacción laboral general.

$* * p<0.01$.

Fuente: elaboración propia.

modelo completo con el ánimo de observar la intensidad de las demás relaciones. Se siguió entonces el segundo paso de verificación de la mediación, a saber, examinar si existían relaciones significativas entre las variables independientes y la mediadora (Baron \& Kenny, 1986) (Figura 2).

Flexibilidad y apertura al cambio mostraron una relación significativa con la satisfacción. No lo hizo el manejo del fracaso.

El siguiente paso consistió en revisar que la variable mediadora se relacionara con las dependientes (Figura 3). Los resultados revelan que la satisfacción laboral se asocia con el cumplimiento de normas y con el desempeño extrarrol, no con el intrarrol.

Se procedió luego a la verificación del modelo de investigación. Según los resultados previos, se examinó si la satisfacción laboral general media la relación entre la apertura al cambio y el desempeño extrarrol. Las demás relaciones han quedado descartadas por no cumplir los supuestos de la mediación. No se confirma la mediación total. El efecto de la apertura al cambio sobre el desempeño extrarrol no desaparece al poner en medio la satis- facción laboral (Figura 4). El cambio del efecto no es significativo según lo muestran los resultados del Test de Sobel $(z$-value $=1.53, p>0.05)$ (Baron $\&$ Kenny, 1986). Se encuentra entonces una mediación parcial.

\section{Discusión}

Se obtuvo confirmación parcial de la hipótesis planteada. De las tres dimensiones de CPs exploradas solo apertura al cambio mostró alguna relación con una de las dimensiones del desempeño laboral (desempeño extrarrol). Por su parte, la satisfacción laboral solo reveló asociaciones significativas con el cumplimiento de normas y el desempeño extrarrol. El efecto de la apertura al cambio sobre las conductas extrarrol no desapareció al ubicar la satisfacción laboral como variable mediadora, lo que habla de una mediación parcial.

Esto sugiere que, en el contexto de la investigación, las personas abiertas, que exploran diferentes alternativas y que muestran una actitud positiva ante lo cambiante de la vida, suelen sentirse más satisfechos e involucrarse con actividades que ex- 


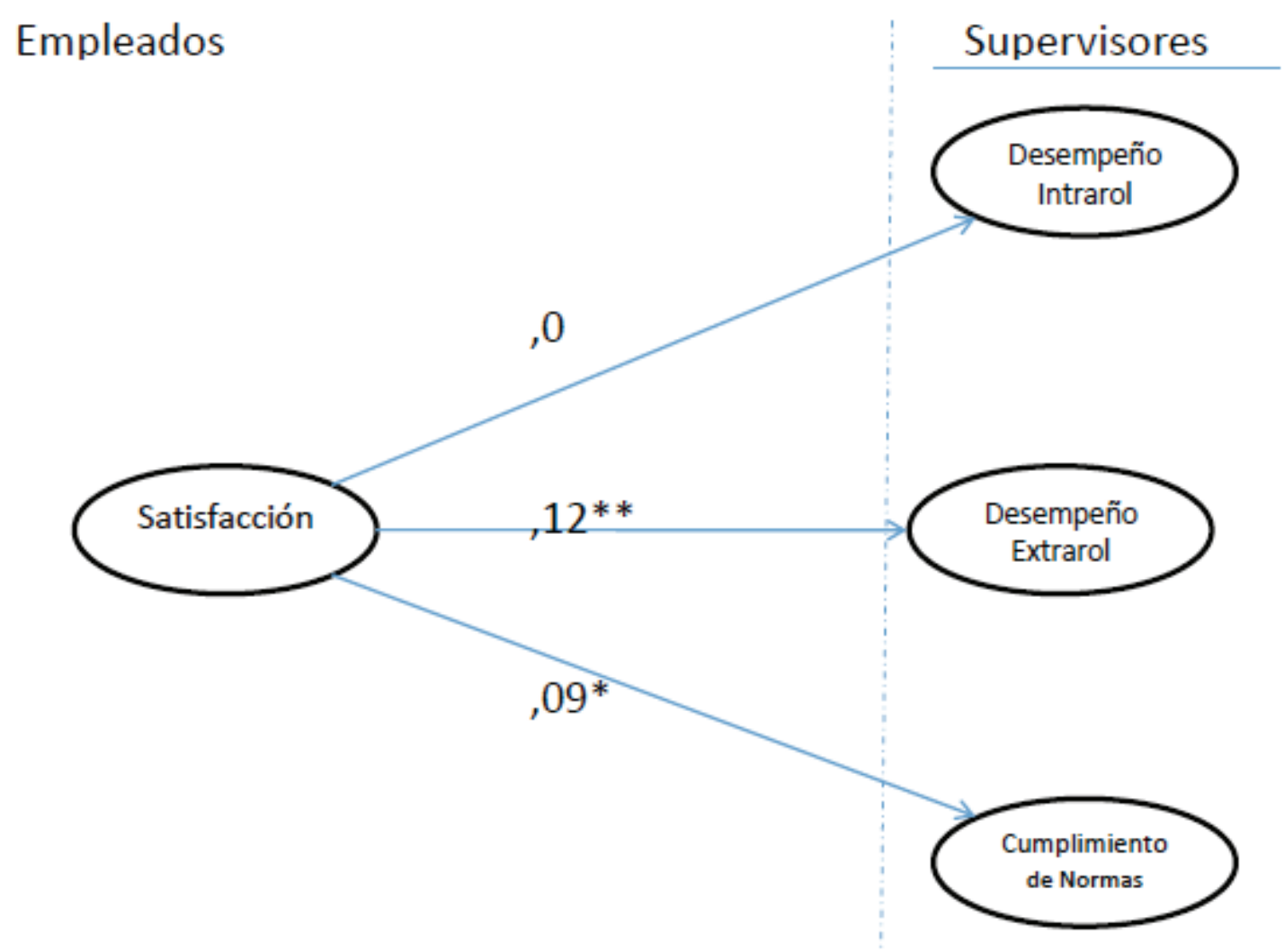

Figura 3. Significación y estimados estandarizados de las relaciones de la satisfacción laboral general con las Dimensiones de desempeño laboral.

$* * * p<0.001$. ** $p<0.01$.

Fuente: elaboración propia.

ceden los límites de su cargo. Esto es consistente con la idea de que quienes se cierran a los cambios suelen experimentar insatisfacción laboral e irritación (Wanberg \& Banas, 2000). Es posible, además, que la relación entre estas variables se deba a que los jefes consideren que los colaboradores abiertos al cambio son personas que agregan valor a su trabajo (Omar \& Uribe, 2005). También es probable que se deba a que ambas son condiciones asociadas con la proactividad (Omar \& Uribe, 2005). Este resultado podría explicarse también por algunas características de la muestra. Esta está compuesta, en su mayoría, por personas con un nivel académico de bachillerato y tecnológico y con edades inferiores a los 30 años. Se trata de un grupo de población más joven y con más posibilidades de desarrollo, lo cual los puede caracterizar como más abiertos al cambio y como personas que consideran que tienen que ofrecer un plus a la organización de sus empresas (Homan, van Knippenberg, van Kleef \& de Dreu, 2007).

La relación de la flexibilidad y la apertura al cambio con la satisfacción laboral sugiere que la capacidad de adaptarse y de abrirse a nuevas experiencias puede llevar a las personas a juzgar más positivamente sus realidades del trabajo y con esto a sentirse más satisfechos, lo cual es consistente con hallazgos previos (Morales de Romero, 2008). Es posible que por tratarse de un proceso de cambio en el cual se busca integrar la realidad exterior e interior desde un punto de vista positivo, experimentado el cambio y la incertidumbre como elementos de CPs (Eljuri, 1994; Salom \& Romero, 1994) se logre una alteración de las expectativas o de las condiciones del entorno haciendo que el trabajo se vuelva más satisfactorio. Es posible que de ahí se derive la 


\section{Empleados}

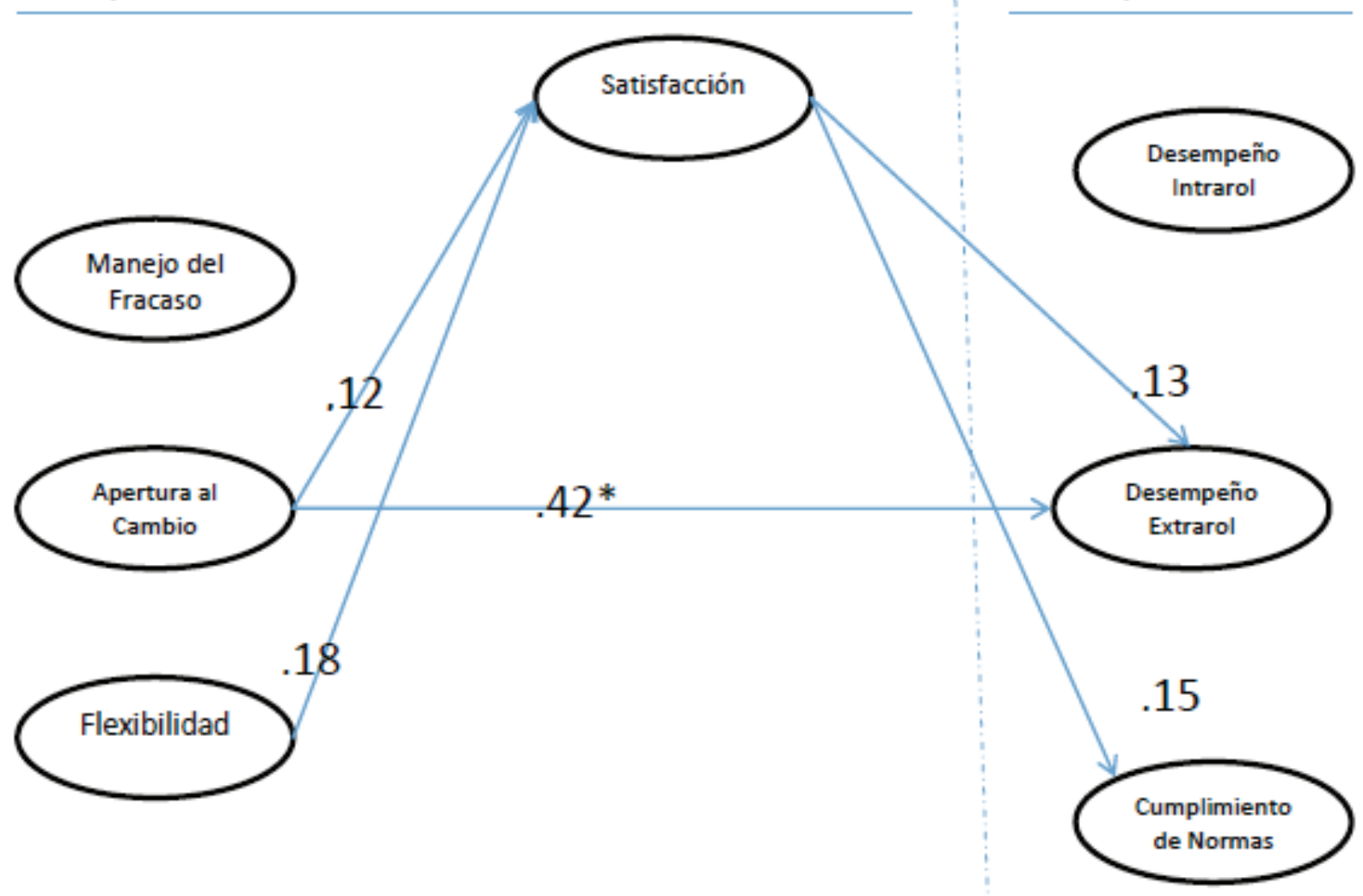

Figura 4. Significación y estimados del modelo de mediación de la satisfacción laboral. ${ }^{* *} p<0.001 . * * p<0.01$. Nota. Se conservan solo los Paths significativos con el ánimo de que el esquema resulte más claro. Fuente: elaboración propia.

relación entre esta característica y la salud mental (Bond et al., 2008). Nuevamente, las condiciones sociodemográficas de los sujetos que participaron en el estudio pueden explicar este tipo de resultado en la medida que, como se explicó, las personas más jóvenes suelen ser más abiertas al cambio, en tanto se encuentran en un proceso de exploración que les permite cambiar más fácilmente sus expectativas (Homan et al., 2007; Lisbona, 2004).

De otra parte, aunque la literatura sugiere que una visión positiva del fracaso podía mejorar la satisfacción (Romero, 1994, 1999), los resultados no apoyan esta premisa. Esto se puede explicar en la medida en que, aunque se vea el fracaso positivamente (Newton et al., 2008) y se le considere útil para el éxito (Polivy \& Herman, 2002), esta sigue siendo una experiencia que se vive como antagónica a la felicidad. Difícilmente, alguien se sentirá satisfecho por fracasar, especialmente cuando se trata de personal operativo, en un país en el que los jefes poseen dificultades para ofrecer retroalimentación (Toro, 2005), logrando que los errores sean vistos solo desde la perspectiva del fracaso y no del aprendizaje. Tampoco se encontró una asociación de esta dimensión del CPs con el desempeño, lo cual puede deberse a que la sola visión positiva del fracaso no garantiza un cambio comportamental.

Por su parte, aunque flexibilidad no se relacionó con ninguna de las dimensiones del desempeño, sí lo hace con cumplimiento de normas de manera indirecta, vía Satisfacción. En este caso la satisfacción es un mecanismo psicológico que explica las relaciones entre otras variables.

La falta de asociación directa de la flexibilidad y el manejo del fracaso con las dimensiones de Desempeño Laboral podría explicarse porque es- 
tos atributos del CPs no producen cambios sobre condiciones antecedentes del desempeño como la motivación o las habilidades (Toro, 2002). Es posible que el manejo del fracaso afecte el desempeño hacia futuro, justo en el momento en el que el aprendizaje derivado de este se traduce a la práctica (Kirkpatrick \& Kirkpatrick, 2009) o cuando los jefes contribuyen a verlo así, especialmente en personas que se encuentran en los primeros años de trabajo y en niveles jerárquicos bajos, como los que caracterizan a la muestra de este estudio. Esta, por lo demás, suele ser demandante de aprecio y afecto por parte de sus superiores (Toro, 1996). Por su parte, la flexibilidad podría afectar el desempeño intrarrol en contextos y cargos donde esta sea una característica exigida por la organización, pues se sabe que una habilidad o competencia solo se desarrolla y evidencia si el contexto lo exige (Spencer, McClelland \& Spencer, 1990). Si bien son muchas las ocasiones en las que las personas deban enfrentar la incertidumbre (Taborda, 2010) y se requiere de flexibilidad por estar en un contexto de cambio permanente, se trata todavía de atributos que se demandan más en personas con responsabilidad de mando en la organización (Sanín, 2007). Es decir que una persona con una flexibilidad alta y un buen manejo de la incertidumbre podría mejorar o no su desempeño, dependiendo del nivel en que su tarea se lo exija. Este es un tema interesante de investigación, pero que escapa a los propósitos del presente estudio.

Otro resultado nos muestra que la satisfacción de las personas puede motivarlas a dar la milla extra (Omar \& Uribe, 2005) y a mostrarse coherentes con las normas y principios definidos por la organización, pero no necesariamente a un mejor desempeño intrarrol (Wright \& Cropanzano, 2000). Según esto, es posible que una persona insatisfecha haga bien su trabajo y que, por el contrario, una satisfecha lo haga de manera pobre. La satisfacción podrá afectar el desempeño de rol, solo si se transforma en un elemento motivacional (Lock \& Lathan, 1990).

Los resultados de esta investigación aportan conocimiento en relación con aspectos positivos del trabajo y de la vida de las personas, y a la com- prensión sobre los antecedentes y las consecuencias de factores psicosociales, además muestra algunas variables que anteceden el desempeño y la satisfacción de las personas. Otorga, además, soporte a investigaciones previas que muestran los efectos de la satisfacción sobre las conductas extrarrol e intrarrol y revela una nueva categoría de desempeño sobre la que tiene efectos, el cumplimiento de normas.

Desde el punto de vista práctico, estos hallazgos muestran que las organizaciones interesadas en mejorar la satisfacción de su personal y, con esto, reducir sus efectos negativos (rotación, ausentismo, quejas del personal,...) deberán apuntar al desarrollo de la flexibilidad y la apertura al cambio de su personal. El hallazgo resulta fundamental para las empresas en general, y especialmente para las colombianas, en tanto les permite entender sobre qué aspectos hacer gestión para conseguir reducciones de la rotación y el ausentismo del personal, dos problemas comunes que ocasionan pérdidas económicas considerables (Littlewood, 2008). En este sentido, la comprensión de estos fenómenos aporta información que sirve de insumo para plantear políticas y estrategias que favorezcan la existencia de condiciones de trabajo saludables. Lo cual es muy necesario en el contexto actual, en el que los costos asociados con la salud en el trabajo son altísimos (Gómez, 2007).

Resultará clave que se busque que las personas estén abiertas al cambio. Evitar el trabajo rutinario y dar participación a los colaboradores, motivarlos para encontrar nuevas y mejoradas formas de hacer las cosas. De esta investigación se deriva entonces que las dimensiones del CPs requieren ser tenidas en cuenta a la hora de diseñar estrategias de intervención de la satisfacción laboral; también cuando se quiere analizar y promover las conductas extrarrol de las personas.

La investigación aporta información sobre el comportamiento de las dimensiones del CPs, el rol mediador de la satisfacción y los antecedentes del desempeño intrarrol, extrarrol y el cumplimiento de normas. Futuras investigaciones podrían examinar el modelo diferenciando por tipo de cargo, ya que es posible que los efectos de estos atributos sobre el desempeño estén en función de esta condición. 
Dada la poca atención que ha recibido el CPs en la literatura científica, explorar su relación, antecedentes y consecuencias sobre otras variables individuales, sociales, organizacionales y extralaborales aportará a la comprensión del fenómeno.

Por lo pronto, conviene señalar que una visión positiva del fracaso no afecta la satisfacción, pero si el aprendizaje. La flexibilidad contribuye a que las personas estén más complacidas con sus realidades de trabajo, y la apertura al cambio cumple un doble papel al asociarse con la satisfacción y apuntar a su desempeño extrarrol. Lo anterior indica que si las organizaciones desean personas que den la milla extra y que cumplan con las normas definidas, es necesario que se preocupen por que el personal esté satisfecho y abierto al cambio. Para dimensionar este trabajo, solo se requiere preguntar: ¿qué empresas, colombianas o extranjeras, no están interesadas en trabajadores que den más y que se adapten a las normas y políticas definidas? Este trabajo de investigación es una clave para saber cómo conseguirlo.

\section{Referencias}

Baron, R. M. \& Kenny, D. A. (1986). The moderatormediator variable distinction in social psychological research: Conceptual, strategic and statistical considerations. Journal of Personality and Social Psychology, 51(6), 1173-1182.

Bond, F., Flaxman, P. \& Bunce, D. (2008). The influence of psychological flexibility on work redesign: Mediated moderation of a work reorganization intervention. Journal of Applied Psychology, 93(3), 645-654.

Elaine, D., Pulakos, E., Arad, S., Donovan, M. \& Plamondon, K. (2000). Adaptability in the workplace: Development of a taxonomy of adaptive performance. Journal of Applied Psychology, 85(4), 612-624.

Eljuri, M. (1994). Motivaciones sociales y crecimiento psicológico en trabajadores industriales. Revista Interamericana de Psicología Ocupacional, 13(2), $107-115$

Gómez, I. (2007). Salud laboral: una revisión a la luz de las nuevas condiciones del trabajo. Universitas Psychologica, 6(1), 105-113.
Homan, A., van Knippenberg, D., van Kleef, G. \& de Dreu, C. (2007). Bridging faultlines by valuing diversity: Diversity beliefs, information elaboration, and performance in diverse work groups. Journal of Applied Psychology, 92(5), 1189-1199.

Judge, T. \& Bono, J. (2001). A Rose by any other name. Are self-esteem, generalized self-efficacy, neuroticism an locus of control indicators of common construct? In B. W. Robert \& R. Hogan (Eds.), Personality psychology in the work place. Washington: APA.

Jöreskog, K. G. \& Sörbom, D. (1986). LISREL user guide version VI (4a. ed.). Mooresville, NC: Scientific Software International.

Kaiser, B. \& DeVries, K. (2010). Introduction to the special issue on developing flexible and adaptable leaders for an age of uncertainty. Consulting Psychology Journal, 62(2), 77-80.

Kirkpatrick, J. \& Kirkpatrick, W. (2009). The Kirkpatrick Model: Past, present and future. Chief Learning Officer, 8(11), 20-55.

Lisbona, A. (2004). La diversidad en las organizaciones. En F. J. Palací (Dir.), Psicología de las organizaciones (pp. 279-300). Madrid: Pearson Prentice.

Littlewood, H. F. (2008). Evitación del trabajo, satisfacción en el trabajo y bienestar emocional: potenciales consecuencias de prácticas gerenciales y la percepción de justicia organizacional. En J. F. Uribe (Ed.), Psicología de la salud ocupacional en México. México: UNAM.

Lock, E. \& Latham, G. (1990). Work motivation. The high performance cycle. New Jersey: Lea.

McGraw, K. O. \& Wong, S. P. (1996). Forming inferences about some intraclass correlation coefficients. Psychological Methods, 1(1), 30-46.

Morales de Romero, N. (2008). La libertad para crecer y preferencias de liderazgo. Revista Interamericana de Psicología Ocupacional, 27(2), 87-102.

Muchinsky, P. (2002). Psicología aplicada al trabajo (6a. ed.). México: Thompson Editores.

Newton, N., Khanna, C. \& Thompson, J. (2008). Workplace failure: Mastering the last taboo. Consulting Psychology Journal: Practice and Research, 60(3), 227-245.

Omar, A. \& Uribe, H. (2005). Las dimensiones de personalidad como predictores de los comporta- 
mientos de ciudadanía organizacional. Estudios de Psicología, 10(2), 157-166.

Podsakoff, P. M., Mackenzie, S. B., Lee, J. \& Podsakoff, N. P. (2003). Common method biases in behavioural research: A critical review of the literature and recommended remedies. Journal of Applied Psychology, 88(5), 879-903.

Polivy, J. \& Herman, P. (2002). If at first you don't succeed false hopes of self-change. American Psychologist, 57(9), 677-689.

Romero, O. (1994). Crecimiento psicológico y motivaciones sociales. Revista Interamericana de Psicología Ocupacional, 13(2), 93-106.

Romero, O. (1999). Crecimiento psicológico y motivaciones sociales (3a. ed.). Mérida: Rogya.

Salom, C. \& Romero, O. (1994). Crecimiento psicológico y motivaciones sociales en trabajadores industriales. Revista Interamericana de Psicología Ocupacional, 13(2), 107-116.

Sanín, A. (2007). Estudio de diferencias en apoyo del jefe e imagen gerencial asociadas al género, nivel educativo y nivel jerárquico. Revista Interamericana de Psicología Ocupacional, 26(2), 38-62.

Sanín, A. (2010). Validación psicométrica de un conjunto de instrumentos que miden factores psicosociales disposicionales. Revista Interamericana de Psicología Ocupacional, 29(2), 146-173.

Sanín, A. \& Restrepo, M. (2009). Vinculación laboral, motivación y desempeño de empleados de carrera y supernumerarios en una universidad del Estado. Revista Interamericana de Psicología Ocupacional, 28(2), 149-183.

Shelley, T. \& Silvia, P. (2002). Self-awareness, probability of improvement, and the self-serving bias. Journal of Personality and Social Psychology, 82(1), 49-61.
Spencer, L., McClelland, D. \& Spencer, S. (1990). Competency assessment methods: History and state of art. Boston: Hay/MCber Research Press.

Taborda, H. (2010). Modelos bayesianos de inferencia psicológica: icómo predecir acciones en situaciones de incertidumbre? Universitas Psychologica, 9(2), 495-507.

Toro, F. (2002). Desempeño y productividad. Medellín: Cincel.

Toro F. (2005). Liderazgo organizacional objeciones y quejas sobre el apoyo del jefe. Revista Interamericana de Psicología Ocupacional, 24(1-2), 46-59.

Toro, F. (2010). Validación psicométrica de un conjunto de instrumentos que evalúan efectos individuales de diversos factores psicosociales. Revista Interamericana de Psicología Ocupacional, 29(2), 193-225.

Toro, F., Londoño, M. E., Sanín, A. \& Valencia, M. (2010). Modelo analítico de factores psicosociales en contextos laborales. Revista Interamericana de Psicología Ocupacional, 29(2), 95-137.

Wanberg, C. \& Banas, J. (2000). Predictors and outcomes of openness to changes in a reorganizing workplace. Journal of Applied Psychology, 85(1), 132-142.

Wright, T. A. \& Cropanzano, R. (2000). Psychological well-being and job satisfaction as predictors of job performance. Journal of Occupational Health Psychology, 5(1), 84-94.

Zelenski, J., Murphy, S. \& Jenkins, D. (2008). The happy-productive worker thesis revisited. Journal of Happiness Studies, 9(4), 521-537. doi:10.1007/ s10902-008-9087-4 
